\title{
Valoración de la cuenca del río Guadiamar (sur de España), afectada por un vertido minero, en base a su odonatofauna.
}

\author{
Manuel Ferreras-Romero, Francisco Jesús Cano-Villegas y Juan César Salamanca-Ocaña \\ Departamento de Ciencias Ambientales (Zoología), Universidad Pablo de Olavide, Ctra. de Utrera km 1, \\ 41013 Sevilla (España - Spain). E-mail: <ferreras@teleline.es>
}

\begin{abstract}
RESUMEN
En este trabajo fue analizada la comunidad de odonatos existente en la parte de la cuenca del río Guadiamar afectada por el vertido minero ocurrido en abril de 1998. El número de especies encontradas (18) no fue particularmente bajo en comparación con otras cuencas andaluzas, pero más de la mitad de ellas pertenecen a la familia Libellulidae (55.5\%). Es significativa la ausencia de especies pertenecientes a familias con un carácter más típicamente reófilo (p. ej. Gomphidae y Calopterygidae), habitualmente presentes en cuencas ibéricas; lo que puede ser considerado un reflejo evidente del mal estado actual de esta parte de la cuenca. En el análisis zoogeográfico destacó el alto porcentaje de elementos norteafricanos.
\end{abstract}

Palabras clave: Odonatos, sur de España, zoogeografía, vertido minero.

\begin{abstract}
ABSTRAT
We analysed the Odonata community in the area of the river Guadiamar catchment (southern Spain) affected by a mining spill which occurred in April 1998. Compared to other Andalusian catchments, the number of species found (18) was not particulary low, although a significant proportion (55.5\%) belonged to the family Libellulidae. Absence of some species common in Iberian rivers belonging to typically rheophilous families (e.g. Gomphidae and Calopterygidae) was significant. This fact highlights the current bad ecological conditions of this part of the catchment. In addition, zoogeographical analysis showed that a high proportion of elements are of North-African origin.
\end{abstract}

Keywords: Odonata, southern Spain, zoogeography, mining spill.

\section{INTRODUCCIÓN}

La cuenca del río Guadiamar ocupa la parte más occidental de la provincia de Sevilla, tiene su origen en Sierra Morena, cerca de la localidad del Castillo de las Guardas, y algunos de los tributarios por la margen derecha nacen en la provincia de Huelva. Desemboca en el Brazo de la Torre del río Guadalquivir, en las proximidades del Parque Nacional de Doñana. Gran parte de esta cuenca es utilizada desde hace muchos años como terrenos agrícolas. Tras la rotura y vertido de la balsa minera de Aznalcóllar en abril de 1998, han tenido lugar diversas actuaciones en el cauce y márgenes para devolver a la zona afectada su situación original, y con el objetivo a largo plazo de alcanzar unas condiciones ecológicas óptimas: diseño de un corredor verde que una Doñana con Sierra Morena. Pero el estado de esta cuenca en su conjunto parece distar mucho de lo que serían sus condiciones naturales originales (Prat et al., 1999, 2001), y son numerosos los estudios que demuestran que elementos tales como la vegetación de ribera no sólo condiciona, por ejemplo, la temperatura del agua, sino que, cuando no ha sido eliminada, impide la llegada de sedimentos a los arroyos. Las prácticas agrícolas en las cuencas fluviales condicionan fuertemente la estructura de las comunidades de macroinvertebrados, ya que la diversidad disminuye a la vez que aumenta el grado de desforestación (Sponseller et al., 2001).

Los insectos del orden Odonatos, con fases de huevo y larva desarrolladas en el medio acuático, 
excelentes voladores en su fase adulta, condición de depredadores a lo largo de toda su vida, y considerados por diversos autores como indicadores de las condiciones generales de los medios acuáticos en que viven (Corbet, 1999), aparecen $a$ priori como organismos muy adecuados para realizar un seguimiento de la evolución de una cuenca que ha experimentado una alteración de enormes dimensiones como es el caso de la aquí tratada, y a la que las Administraciones nacional y autonómica están destinando cuantiosos fondos. En el conjunto de las especies de este orden, el grado de tolerancia/exigencia a numerosos factores físicos y químicos es muy variable, como han puesto de manifiesto numerosos autores (Roback, 1974; Carchini \& Rota, 1985; Ferreras-Romero, 1988). El presente trabajo estudia la composición de las comunidades de odonatos existentes en la zona afectada por el vertido (transcurridos dos años del mismo), comparándolas con las existentes en puntos de la cuenca próximos pero no afectados (Fig. 1). Con esto se pretende cuantificar el estado actual de la parte media-inferior del Guadiamar, en función de las especies de estos insectos que alberga, y plantear hipótesis acerca de la posible evolución de la zona afectada.

\section{MATERIAL Y MÉTODOS}

Tras un muestreo preliminar realizado en abril de 2000 fueron seleccionadas ocho localidades: cuatro en la parte del eje principal afectada por el vertido, a las que consideramos "sumideros"; una en el eje principal de la cuenca, aguas arriba de la zona del vertido, y tres arroyos no afectados, que drenan a tramos del río por los que circularon los lodos tóxicos; estas últimas cuatro localidades

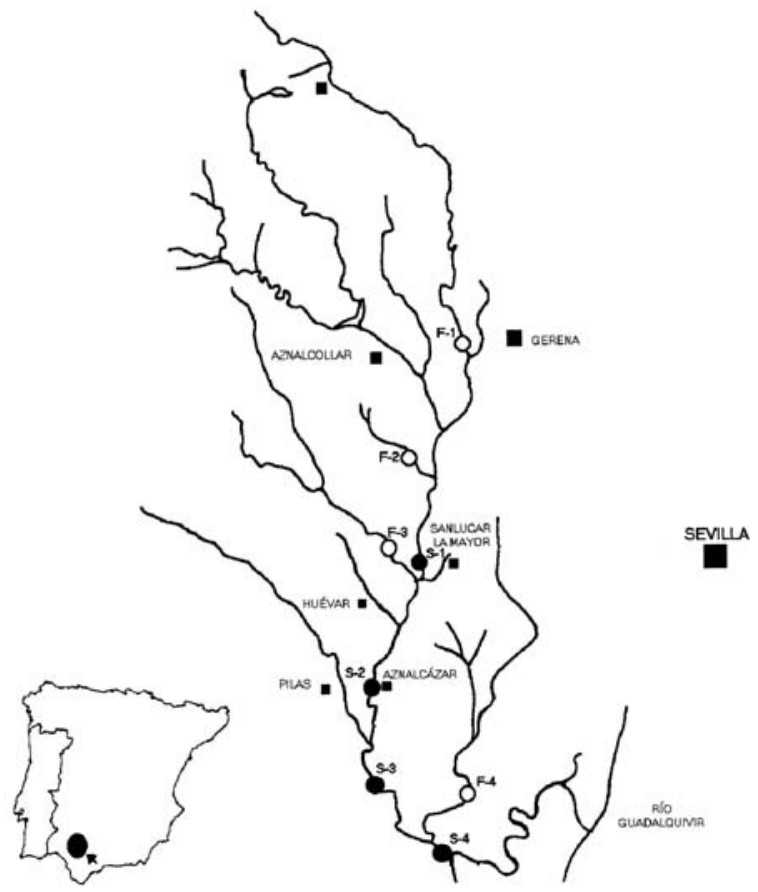

Figura 1. Localización del área estudiada en la península ibérica. Se indican los lugares de la cuenca donde se muestreó mensualmente desde abril hasta septiembre de 2000 (círculos vacíos, F-1, 2, 3 y 4, localidad del curso principal y arroyos no afectados por el vertido minero; círculos llenos, S-1, 2, 3 y 4, localidades de los tramos medio y bajo del río afectados por el vertido). Location of the study area on the Iberian Peninsula. The sites in the catchment are indicated where dragonflies were collected monthly from April to September 2000 (empty circles, $F-1,2,3$ and 4, are sites of the main channel and tributaries which were not affected by the mining spill; filled circles, $S-1,2$, 3 and 4 , are sites in middle and lower reaches of the main channel which were affected by the spill).

fueron consideradas como posibles "fuentes" de recolonización del tramo afectado (Tabla 1). En estas ocho localidades fueron recolectados mensualmente odonatos adultos, desde abril hasta septiembre del año 2000.

Tabla 1. Descripción de los puntos de muestreo y coordenadas geográficas. Description of the sampling points and geographical coordinates.

\begin{tabular}{llll}
\hline No afectadas por el vertido & Coordenadas UTM & Afectadas por el vertido & Coordenadas UTM \\
\hline F-1, río Guadiamar - Gerena & 29S QB 4857 & S-1, río Guadiamar - Sanlúcar la Mayor & 29S QB 4542 \\
F-2, arroyo Las Charcas, & 29S QB 4547 & S-2, río Guadiamar - Aznalcázar & 29S QB 4332 \\
F-3, arroyo Ardachón, & 29S QB 4342 & S-3, río Guadiamar - vado de Quemas & 29S QB 4225 \\
F-4, arroyo Majaberraque & 29S QB 4923 & S-4, río Guadiamar - vado de Don Simón & 29S QB 4818 \\
\hline
\end{tabular}


Tabla 2. Valores de abundancia (según Dufour, 1978: 1, observación de un individuo; 2 , hasta cuatro $\left(2^{2}\right)$ individuos; 3 , hasta ocho $\left(2^{3}\right)$ individuos ...); e información adicional de las localidades no afectadas por el vertido minero (fuentes): E, recogida de exuvias; R, observación de actividad reproductora (cópula, tándem, oviposición); T, captura de ejemplares recién emergidos (teneral). Dufour abundances (Dufour, 1978) and additional observations in sites not affected by the mining spill (sources): E, presence of exuviae; $R$, reproduction activity recorded (copulation, tandem, oviposition); $T$, collection of teneral adults.

\begin{tabular}{|c|c|c|c|c|c|c|c|c|c|c|c|c|}
\hline & \multicolumn{4}{|c|}{ Abril } & \multicolumn{4}{|c|}{ Mayo } & \multicolumn{4}{|c|}{ Junio } \\
\hline & F-1 & $\mathrm{F}-2$ & F-3 & F-4 & F-1 & $\mathrm{F}-2$ & F-3 & F-4 & F-1 & $\mathrm{F}-2$ & $\mathrm{~F}-3$ & F-4 \\
\hline Lestes viridis & - & - & - & - & $2 \mathrm{~T}$ & - & - & $3 \mathrm{~T} \mathrm{E}$ & - & - & - & - \\
\hline Platycnemis latipes & - & - & - & - & - & - & - & - & $4 \mathrm{R}$ & - & - & - \\
\hline Platycnemis acutipennis & - & - & - & - & $2 \mathrm{~T}$ & 1 & $2 \mathrm{R}$ & - & 2 & - & - & - \\
\hline Cercion lindenii & - & - & - & - & - & - & 1 & - & 2 & - & - & - \\
\hline Ischnura graellsii & - & - & $3 \mathrm{~T}$ & 3 & $5 \mathrm{~T}$ & 2 & & $4 \mathrm{R}$ & $5 \mathrm{R}$ & 3 & 2 & 5 \\
\hline Anax parthenope & - & - & - & - & - & - & - & - & - & - & - & - \\
\hline Orthetrum chrysostigma & - & - & - & - & $1 \mathrm{~T}$ & - & - & - & 2 & $2 \mathrm{~T}$ & $2 \mathrm{R}$ & $3 \mathrm{R}$ \\
\hline Orthetrum cancellatum & - & - & - & - & $1 \mathrm{~T}$ & - & - & - & - & - & - & - \\
\hline Orthetrum coerulescens & - & - & - & - & - & - & - & - & - & - & - & - \\
\hline Diplacodes lefebvrii & - & - & - & - & - & - & - & - & - & - & - & - \\
\hline Brachythemis leucosticta & - & - & - & - & - & - & - & - & - & - & - & - \\
\hline Crocothemis erythraea & - & - & - & - & - & 1 & - & - & 1 & 3 & - & $3 \mathrm{R}$ \\
\hline Sympetrum striolatum & - & - & - & - & $2 \mathrm{~T}$ & 2 & - & - & - & - & 2 & - \\
\hline Sympetrum fonscolombii & - & - & - & - & - & - & - & - & 3 & 1 & - & $3 \mathrm{~T}$ \\
\hline \multirow[t]{3}{*}{ Trithemis annulata } & - & - & - & - & - & - & - & - & 3 & - & - & - \\
\hline & \multicolumn{4}{|c|}{ Julio } & \multicolumn{4}{|c|}{ Agosto } & \multicolumn{4}{|c|}{ Septiembre } \\
\hline & F-1 & F-2 & F-3 & F-4 & F-1 & F-2 & F-3 & F-4 & F-1 & $\mathrm{F}-2$ & F-3 & F-4 \\
\hline Lestes viridis & - & - & - & - & - & - & - & - & - & - & - & - \\
\hline Platycnemis latipes & 3 & - & - & - & - & - & - & - & - & - & - & - \\
\hline Platycnemis acutipennis & - & - & - & - & - & - & - & - & - & - & - & - \\
\hline Cercion lindenii & - & - & - & - & - & - & - & - & - & - & - & - \\
\hline Ischnura graellsii & 5 & $3 \mathrm{R}$ & 2 & $4 \mathrm{R}$ & $2 \mathrm{~T}$ & 1 & $3 \mathrm{R}$ & $4 \mathrm{R}$ & $2 \mathrm{R}$ & - & 2 & 4 \\
\hline Anax parthenope & - & - & - & - & $2 \mathrm{R}$ & - & - & 2 & - & - & - & - \\
\hline Orthetrum chrysostigma & 2 & 1 & - & - & 1 & 2 & - & - & 2 & - & - & \\
\hline Orthetrum cancellatum & - & - & - & - & 2 & - & - & 3 & - & - & - & 2 \\
\hline Orthetrum coerulescens & 1 & - & - & - & - & - & - & - & - & - & - & - \\
\hline Diplacodes lefebvrii & - & - & - & - & - & - & - & 1 & - & - & - & - \\
\hline Brachythemis leucosticta & - & - & - & - & $1 \mathrm{~T}$ & - & - & - & 2 & - & - & - \\
\hline Crocothemis erythraea & - & 2 & 2 & 1 & 3 & 3 & $4 \mathrm{R}$ & 3 & 1 & 2 & 2 & $2 \mathrm{~T}$ \\
\hline Sympetrum striolatum & - & - & - & - & - & - & - & - & - & - & - & - \\
\hline Sympetrum fonscolombii & $3 \mathrm{R}$ & 4 & 3 & 7 & $3 \mathrm{R}$ & $3 \mathrm{TR}$ & $4 \mathrm{~T}$ & $4 \mathrm{R}$ & $4 \mathrm{R}$ & 2 & 4 & $4 \mathrm{TR}$ \\
\hline Trithemis annulata & 2 & - & - & - & 3 & - & - & - & 3 & - & - & - \\
\hline
\end{tabular}

La toma de datos en cada localidad fue realizada durante sesenta minutos, aproximadamente, a las horas centrales del día, entre las 12 y las 17, hora oficial en España, que es cuando tiene lugar la actividad propia de los adultos (defensa de territorio, cópulas, puestas), a fin de garantizar la detección de un número de especies coincidente o muy próximo al real en los diferentes lugares de muestreo. Los ejemplares capturados, tras ser marcados con rotuladores indelebles e identificados in situ, fueron liberados; sólo un escaso número de ellos fue fija- do en alcohol (70\%) para su posterior determinación en el laboratorio. Para realizar estimas de abundancia se ha seguido el criterio de Dufour (1978). También fueron anotadas las observaciones de cópulas y puestas, así como los individuos de emergencia reciente (tene$\mathrm{ral}$ ). Asimismo, fueron recogidas (alcohol $70 \%$ ) e identificadas en el laboratorio las exuvias encontradas en la vegetación de orilla.

Como base del análisis zoogeográfico fueron utilizadas las categorías propuestas por St. Quentin (1960) y por Ocharan-Larrondo (1988). 


\section{RESULTADOS}

Fueron identificados ejemplares de dieciocho especies, pertenecientes a cinco familias: Libelúlidos fue la mejor representada. Quince especies fueron observadas en puntos no afectados por el vertido y catorce en el conjunto de tramos del río por donde pasaron los lodos tóxicos; once especies fueron vistas en ambos tipos de localidades. Su relación, ordenada según Askew (1988), aparece a continuación. Ninguno de los ejemplares marcados durante este estudio fue recapturado con posterioridad.

\section{LESTIDAE}

Lestes viridis (Van der Linden, 1825)

Especie con distribución holomediterránea (St. Quentin, 1960; Ocharan-Larrondo, 1988), habitualmente asociada a cursos temporales o con grandes fluctuaciones de nivel (Castella, 1987; Ferreras-Romero \& García Rojas, 1995; AgüeroPelegrín et al., 1999). Sólo fue hallada en dos localidades, ambas consideradas "fuentes", el río Guadiamar a su paso cerca de Gerena y el arroyo Majaberraque; en éste fueron encontradas dos exuvias. Los valores de abundancia son bajos en ambas localidades (Tabla 2).

Tabla 3. Valores de abundancia e información adicional de las localidades afectadas por el vertido minero (sumideros); abreviaturas como en Tabla 2. Abundances and additional observations in sites affected by a mining spill (sinks). Abbreviations as for Table 2.

\begin{tabular}{|c|c|c|c|c|c|c|c|c|c|c|c|c|}
\hline & \multicolumn{4}{|c|}{ Abril } & \multicolumn{4}{|c|}{ Mayo } & \multicolumn{4}{|c|}{ Junio } \\
\hline & S-1 & S-2 & S-3 & S-4 & S-1 & S-2 & S-3 & S-4 & S-1 & $\mathrm{S}-2$ & S-3 & S-4 \\
\hline Platycnemis acutipennis & - & - & - & - & 1 & - & - & - & - & - & - & - \\
\hline Cercion lindenii & - & - & - & - & - & - & - & - & - & - & - & - \\
\hline Enallagma cyathigerum & - & - & 3 & - & - & - & 1 & - & - & - & $2 \mathrm{R}$ & - \\
\hline Ischnura graellsii & - & - & - & - & $4 \mathrm{~T}$ & 1 & 4 & 5 & 3 & $2 \mathrm{~T}$ & $4 \mathrm{R}$ & - \\
\hline Anax parthenope & - & - & - & - & - & - & - & - & - & - & - & - \\
\hline Hemianax ephippiger & - & - & - & - & - & - & - & - & - & - & - & - \\
\hline Orthetrum trinacria & - & - & - & - & - & - & - & - & - & - & - & - \\
\hline Orthetrum chrysostigma & - & - & - & - & - & $1 \mathrm{~T}$ & - & - & 2 & - & - & - \\
\hline Orthetrum cancellatum & - & - & - & - & - & - & 2 & - & - & 1 & 3 & - \\
\hline Brachythemis leucosticta & - & - & - & - & 1 & - & - & - & 2 & - & - & - \\
\hline Crocothemis erythraea & - & - & - & - & - & - & - & - & 1 & 1 & - & - \\
\hline Sympetrum striolatum & - & - & 2 & - & - & - & - & - & - & - & - & - \\
\hline Sympetrum fonscolombii & - & - & - & - & $3 \mathrm{~T}$ & 1 & - & - & $4 \mathrm{R}$ & $3 \mathrm{R}$ & 3 & 4 \\
\hline \multirow[t]{3}{*}{ Trithemis annulata } & - & - & - & - & - & - & - & - & - & - & - & - \\
\hline & \multicolumn{4}{|c|}{ Julio } & \multicolumn{4}{|c|}{ Agosto } & \multicolumn{4}{|c|}{ Septiembre } \\
\hline & S-1 & $\mathrm{S}-2$ & S-3 & S-4 & S-1 & $\mathrm{S}-2$ & $\mathrm{~S}-3$ & S-4 & S-1 & S-2 & S-3 & S-4 \\
\hline Platycnemis acutipennis & - & - & - & - & - & - & - & - & - & - & - & - \\
\hline Cercion lindenii & 1 & - & - & - & - & - & - & - & - & - & - & - \\
\hline Enallagma cyathigerum & - & - & 1 & - & - & - & - & - & - & - & - & - \\
\hline Ischnura graellsii & 4 & 4 & 2 & $4 \mathrm{R}$ & 3 & $4 \mathrm{R}$ & 4 & 5 & 3 & $2 \mathrm{R}$ & 2 & 4 \\
\hline Anax parthenope & - & - & - & - & 2 & 1 & $3 \mathrm{R}$ & - & - & $2 \mathrm{R}$ & $2 \mathrm{R}$ & - \\
\hline Hemianax ephippiger & - & - & - & $1 \mathrm{E}$ & - & - & - & - & - & - & - & - \\
\hline Orthetrum trinacria & 1 & - & - & - & 1 & 1 & 1 & - & - & 2 & - & - \\
\hline Orthetrum chrysostigma & 2 & - & - & - & $2 \mathrm{R}$ & - & - & - & - & - & - & - \\
\hline Orthetrum cancellatum & 2 & - & - & - & $3 \mathrm{R}$ & 3 & $2 \mathrm{R}$ & 2 & - & 1 & - & - \\
\hline Brachythemis leucosticta & 2 & - & - & - & $5 \mathrm{~T}$ & 2 & 1 & - & 3 & - & 1 & - \\
\hline Crocothemis erythraea & 2 & 2 & 1 & - & 2 & 3 & 2 & $3 \mathrm{~T}$ & 1 & 1 & 3 & $3 \mathrm{~T}$ \\
\hline Sympetrum striolatum & - & - & - & - & - & - & - & - & - & - & - & - \\
\hline Sympetrum fonscolombii & 5 & $5 \mathrm{R}$ & $5 \mathrm{R}$ & $7 \mathrm{R}$ & $4 \mathrm{~T} \mathrm{R}$ & $5 \mathrm{~T}$ & $4 \mathrm{~T} \mathrm{R}$ & $5 \mathrm{~T}$ & 4 & $3 \mathrm{R}$ & $2 \mathrm{R}$ & $5 \mathrm{R}$ \\
\hline Trithemis annulata & 1 & - & - & - & 2 & 2 & 1 & - & 3 & 2 & - & - \\
\hline
\end{tabular}




\section{PLATYCNEMIDIDAE}

Platycnemis latipes Rambur, 1842

Especie considerada holomediterránea por St. Quentin (1960) y de distribución ibero-magrebí por Ocharan-Larrondo (1988). Ferreras-Romero (1999) la incluye entre las trece especies que considera endémicas sensu lato del área mediterránea occidental. Sólo fue encontrada en el río Guadiamar en el término de Gerena, con valores de abundancia aceptables o discretos.

\section{Plactycnemis acutipennis Sélys, 1841}

Reconocida por todos los autores como exclusiva del Mediterráneo occidental, ibero-magrebí (St. Quentin, 1960; Ocharan-Larrondo, 1988). Esta especie fue hallada en tres fuentes, el río Guadiamar a su paso cerca de Gerena, los arroyos de las Charcas y Ardachón, y en un punto afectado por el vertido, el río Guadiamar a su paso por Sanlúcar. Es una especie típica del eje principal de los ríos de Sierra Morena, donde coexiste con la especie anterior. Las observaciones fueron muy escasas o con valores de abundancia discretos (Tablas 2 y 3 ).

\section{COENAGRIONIDAE}

\section{Cercion lindenii (Sélys, 1840)}

Especie ampliamente distribuida por el Mediterráneo occidental (St. Quentin, 1960; Ocharan-Larrondo, 1988). Fue encontrada en tres localidades, siempre en escaso número: el río Guadiamar cerca de Gerena, el arroyo Ardachón y Sanlúcar la Mayor, este último afectado por el vertido minero. Al igual que las dos especies anteriores, suele ser abundante en cursos medios de ríos de Sierra Morena (Ferreras-Romero, 1991; Ferreras-Romero \& García-Rojas 1995).

Enallagma cyathigerum (Charpentier, 1940). Con distribución holoártica, sólo ha sido detectada en un tramo del río Guadiamar afectado por el vertido, el vado del cortijo de Quemas. Los registros de adultos se extienden desde abril hasta julio, de forma ininterrumpida, aunque únicamente fue observada actividad reproductora (tándem) en junio.
Ischnura graellsii (Rambur, 1842).

Especie ampliamente distribuida en la península ibérica, su área de distribución está limitada al extremo del Mediterráneo occidental, tratándose de una especie ibero-magrebí, frecuente y abundante en medios leníticos y lóticos con escasa velocidad de la corriente. Es el único zigóptero que ha sido encontrado en todos los puntos muestreados, y en muchos casos con unos niveles de abundancia medios o aceptables; el valor medio de estos niveles en fuentes y sumideros fue absolutamente similar (2.66, en ambos casos). Se trata de una especie ecológicamente muy tolerante (Ferreras-Romero, 1988) tanto a factores físicos como químicos.

\section{AESHNIDAE}

Anax parthenope (Sélys, 1839)

St. Quentin (1960) la encuadra como autóctona del Mediterráneo oriental, y para OcharanLarrondo (1988) es un elemento póntico-oriental. Fue capturada en cinco localidades, dos consideradas fuentes y tres afectadas por el vertido (Tablas 2 y 3). En todas ellas las observaciones tuvieron lugar a partir del mes de agosto, con valores discretos de abundancia. Se capturó en Gerena, el arroyo Majaberraque (ambos fuentes), y en el río Guadiamar a su paso por Sanlúcar, Aznalcázar y el vado del Quemas.

Hemianax ephippiger (Burmeister, 1839)

Autóctona del mediterráneo oriental (St. Quentin, 1960), o considerada elemento etiópico (Ocharan-Larrondo, 1988). En este estudio sólo fue recogida una exuvia, en el vado de Don Simón; zona afectada por el vertido en el límite norte de la marisma.

\section{LIBELLULIDAE}

\section{Orthetrum trinacria (Sélys, 1841)}

St. Quentin (1960) la considera una especie alóctona con origen en el norte de África, Ocharan-Larrondo (1988) un elemento de origen etiópico. En la península ibérica sólo se conoce en provincias litorales de Andalucía (Huelva, Almería) y en el sur de Extremadura (Badajoz). Fueron vistos ejemplares en tres de los cuatro 
puntos estudiados de la zona afectada por el vertido (Tabla 2), pero nunca fue registrada en los puntos no afectados. El número de ejemplares fue siempre muy escaso.

Orthetrum chrysostigma (Burmeister, 1839)

Encuadrada por los autores mencionados en las mismas categorías que la especie anterior, alóctona con origen en el norte de África y de origen etiópico. Citada por primera vez en Andalucía en el siglo XIX (Hagen, 1866; ver OcharanLarrondo, 1987), en la península ibérica también ha sido citada en el Levante y el sur de Portugal. A diferencia de $O$. trinacria, se trata de una especie con poblaciones numerosas, bien establecidas. Fue hallada en la mayoría de las localidades visitadas (Tablas 2 y 3). Su presencia en las fuentes es más constante que en los sumideros, y la abundancia ligeramente superior. Nunca fue vista en los dos puntos afectados de menor cota, a pesar de que su presencia a principio de verano (junio) en el arroyo Majaberraque, con actividad reproductora incluida, fue notable.

Orthetrum cancellatum (Linneo, 1758)

$\mathrm{Su}$ distribución es holomediterránea (St. Quentin, 1960; Ocharan-Larrondo, 1988). Fue encontrada en todos los puntos afectados por el vertido y en dos no afectados, Gerena y el arroyo Majaberraque. Su presencia en los sumideros fue relativamente constante, registrándose los valores de abundancia más altos y la actividad reproductora en agosto.

\section{Orthetrum coerulescens (Fabricius, 1798)}

Como la especie anterior, su distribución es holomediterránea. A lo largo de todo este estudio sólo fue encontrado un ejemplar: en el río Guadiamar cerca de Gerena, en el muestreo de julio.

\section{Diplacodes lefebvrii (Rambur, 1842)}

Se trata de nuevo de un libelúlido alóctono con origen en el norte de África (Galletti \& Pavesi, 1987), o elemento de origen etiópico (OcharanLarrondo, 1988). Sólo fue recogido un ejemplar en el arroyo Majaberraque. En la península ibérica se conoce en la mitad sur de España y
Portugal, datando las citas más antiguas de la primera mitad del siglo XX.

Brachythemis leucosticta (Burmeister, 1839)

Es otro libelúlido alóctono con origen en el norte de África (Bucciarelli et al., 1983), o elemento de origen etiópico (Ocharan-Larrondo, 1988). Su presencia resulta en los últimos años cada vez más frecuente en el sur peninsular. Fue encontrada en tres de las localidades afectadas por el vertido, y en un punto no afectado, el río Guadiamar cerca de Gerena. En los puntos considerados sumideros su constancia y abundancia fue claramente superior, resultando considerable su número en el mes de agosto en el tramo del río próximo a Sanlúcar la Mayor. Adultos con coloración no definitiva (teneral) fueron observados en las dos localidades mencionadas.

Crocothemis erythraea (Brulle, 1832)

Como en el caso de otras especies anteriores se trata de una especie alóctona con origen en el norte de África, un elemento de origen etiópico. Citada en Andalucía ya en el siglo XIX (Hagen, 1866; ver Ocharan-Larrondo, 1987), en la actualidad hay registros de su presencia en varios países de Europa central (Askew, 1988). Fue hallada en todos los puntos muestreados, con valores de abundancia discretos o medios, ligeramente inferiores en las zonas afectadas por el vertido en relación a aquellas consideradas posibles fuentes de recolonización (Tablas 2 y 3 ).

Sympetrum striolatum (Charpentier, 1840)

Distribución holomediterránea. Sólo fue observada entre abril y junio, en tres localidades no afectadas por el vertido y en el vado del cortijo de Quemas, con valores de abundancia siempre bajos.

Sympetrum fonscolombii (Sélys, 1840)

St. Quentin (1960) considera que esta especie tiene distribución holomediterránea, mientras que Ocharan-Larrondo (1988) la incluye en el grupo de elementos de origen etiópico. Es una de las tres especies encontradas en los ocho puntos estudiados. Su presencia es constante 
desde junio hasta septiembre, tanto en fuentes como en sumideros, con valores de abundancia muy altos en julio en el arroyo Majaberraque y en el vado de Don Simón. Estos dos puntos están muy próximos a los campos de cultivo de arroz de las marismas del Guadalquivir, medio artificial en el que podría completar al menos dos generaciones a lo largo del verano. La abundancia en las localidades afectadas por el vertido es superior (valor medio 3.00) a la registrada en las localidades consideradas fuentes (valor medio 2.16).

Trithemis annulata (Palisot de Beauvois, 1805) Desde finales de los años 70 , viene siendo citada en toda la mitad sur de la península ibérica, e incluso más arriba en la zona atlántica (Portugal) y mediterránea (Levante). En los 90 fue observada por primera vez en Francia continental, en los alrededores de Perpiñán (Grand, 1994). St. Quentin (1960) la considera especie alóctona con origen en el norte de África, Ocharan-Larrondo (1988) un elemento de origen etiópico. Ejemplares de esta especie fueron observados en Gerena y en toda la zona afectada por el vertido, salvo en el vado de Don Simón. La presencia de esta especie es ligeramente superior, en cuanto a abundancia, y más temprana en el tiempo, en el tramo no afectado por el vertido (Tablas 2 y 3 ).

Considerando, a lo largo del tiempo, el número de especies encontradas en el conjunto de "fuentes", por un lado, y de "sumideros", por otro, pudo constatarse que durante los meses de primavera la riqueza es mayor en las fuentes. Sin embargo, a lo largo del verano hay un incremento continuado del número observado en los sumideros, con un máximo en julio (once especies); tendencia que no se produce en las consideradas fuentes (máximos en junio y agosto, nueve especies). En julio, agosto y septiembre el número medio de especies observadas fue mayor en los teóricos "sumideros" que en las "fuentes" (Fig. 2). Cuatro especies sólo fueron observadas en puntos no afectados: $L$. viridis, $P$. latipes, $O$. coerulescens y $D$. lefebvrii. Las registradas exclusivamente en

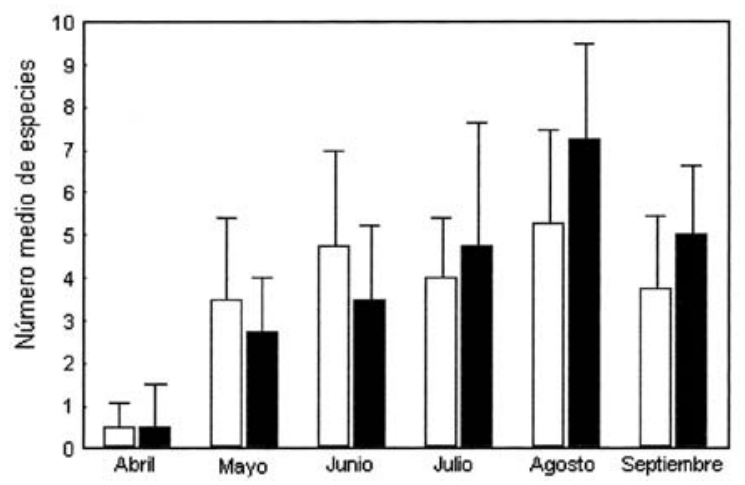

Figura 2. Número medio de especies de odonatos hallados mensualmente (abril-septiembre 2000) en puntos no afectados por el vertido minero (posibles fuentes de recolonización) y en puntos afectados (sumideros). Monthly average of odonata species (April-September 2000) in sites not affected by a mining spill (i.e. potential sources for recolonization), and sites affected by the spill (i.e. sinks).

tramos afectados fueron E. cyathigerum, $H$. ephippiger y O. trinacria. Las especies más frecuentes en esta parte de la cuenca, vistas en los ocho puntos estudiados, fueron I. graellsii, C. erythraea y $S$. fonscolombii.

Tomando como base las categorías zoogeográficas de St. Quentin (1960), revisadas con posterioridad (ver Ferreras-Romero, 1989), en la zona estudiada fueron recogidas seis especies catalogadas como "alóctonas con origen en el norte de África" (66.6\% de las incluidas en dicha categoría) y otras seis consideradas "holomediterráneas" (40.0\%); ambas categorías incluyen dos terceras partes de las especies halladas (Tabla 4). El $80.0 \%$ de las especies catalogadas por Ocharan-Larrondo (1988) como "elementos etiópicos" fueron encontradas en la cuenca; sólo faltan Paragomphus genei (Sélys, 1841) y Zygonyx torridus (Kirby, 1889). Además, algunas de éstas (B. leucosticta, S. fonscolombii) presentan, en determinados meses del año, valores de abundancia que se encuentran entre los más altos registrados en este estudio; sólo alcanzados por el zigóptero I. graellsii, especie catalogada como "elemento ibero-magrebí". 
Tabla 4. Análisis zoogeográfico de las especies presentes en la cuenca del río Guadiamar afectada por el vertido minero y en su entorno inmediato, en relación al conjunto de la odonatofauna andaluza, a partir de las categorías propuestas por St.Quentin (1960). Zoogeographical analysis of species inhabiting the area affected by a mining spill and adjacent areas of the river Guadiamar catchment compared to the general Andalusian odonate fauna. Categories as in St. Quentin (1960).

\begin{tabular}{lcccccc}
\hline $\begin{array}{l}\text { Categorías zoogeográficas } \\
\text { (St.Quentin 1960, }\end{array}$ & \% Andalucía & $\begin{array}{c}\text { Guadiamar } \\
\text { Férea afectada) }\end{array}$ & & & $\begin{array}{c}\text { Guadiamar } \\
\text { (entorno } \\
\text { no afectado) }\end{array}$ & $\begin{array}{c}\text { Guadiamar } \\
\text { área } \\
\text { y entorno } \\
\text { inmediato) }\end{array}$ \\
\hline & & & & & &
\end{tabular}

\section{DISCUSIÓN}

Han aparecido representantes de cinco de las nueve familias presentes en la península ibérica. Es destacable la ausencia, en toda el área estudiada, de especies de las familias Calopterígidos y Gónfidos, mayoritariamente vinculadas a los medios lóticos (Jurzitza, 1993), frecuentes en los ríos que drenan Sierra Morena (FerrerasRomero, 1982; Ferreras-Romero \& GarcíaRojas, 1995), y en el caso de la segunda familia citada, presente en el tramo medio-bajo del río Guadiamar en el pasado reciente (FerrerasRomero \& Gallardo Mayenco, 1985). Tanto en la zona afectada como en el entorno no afectado por el vertido minero, más de la mitad de las especies encontradas fueron libelúlidos: 57.1 y $60.0 \%$, respectivamente. Este hecho ha sido también observado por Prat et al. (2001) al estudiar la comunidad de macroinvertebrados bentónicos. La mayoría de las especies de esta familia no son características de medios fluviales, sino más propias de ambientes leníticos.

La hipótesis de trabajo de considerar posibles fuentes de recolonización aquellos cursos de la parte media y baja de la cuenca no afectados por el vertido resultó poco consistente. El número de especies comunes a zona afectada y no afectada fue de once $(61.1 \%$ de la riqueza en especies registrada), y el número de especies observadas en uno y otro tipo de localidades muy similar, quince (en fuentes) y catorce (en sumideros). La media de especies en las localidades no afectadas coincidió con la media registrada en puntos afectados (8.75 en ambos casos). Mientras que en tres de los hipotéticos sumideros la riqueza observada fue superior a la media (9-11 especies), y sólo en el vado de Don Simón, parte final del Guadiamar que discurre encauzada por zona de marisma dedicada al cultivo de arroz, el número de especies observadas fue muy bajo (5 especies), en tres de las localidades tomadas como fuentes (arroyos de las Charcas, Ardachón y Majaberraque) la riqueza osciló entre 6 y 8 , es decir, inferior a la media de fuentes y de sumideros. Este bajo número de especies en cursos que podrían ser origen de un proceso de recolonización del tramo afectado por el vertido, responde a la intensa actividad agrícola que desde hace muchos años tiene lugar en el entorno de los mismos, con destrucción de la vegetación riparia, fenómenos de intensa erosión y sedimentación, eutrofización de aguas por aportes de nutrientes, contaminación por pesticidas, etc.

La ausencia de especies de la fauna europea típicamente asociada a cursos fluviales, p. ej. las pertenecientes a los géneros Calopteryx, Gomphus u Oxygastra, junto con los datos relati- 
vos a la semejanza en cuanto a riqueza registrada en puntos afectados y no afectados por el vertido minero, y la abundante presencia de especies alóctonas con origen en el norte de África, o de elementos etiópicos, en todos los lugares estudiados, evidencian del mal estado de conservación de la cuenca, tanto en los tramos por los que circularon los lodos tóxicos como en aquellos puntos ubicados en zonas sometidas a una intensa actividad agrícola. Las especies africanas $B$. leucosticta, C. erythraea, S. fonscombii y $T$. annulata son en la actualidad frecuentes y abundantes en medios artificiales con acusadas fluctuaciones de nivel, como embalses destinados a regadíos o a fines recreativos, tramos de río situados aguas abajo de presas, e incluso en estanques urbanos con paredes de hormigón (Agüero-Pelegrín \& Ferreras-Romero, 1992, 1994); prueba de la extrema tolerancia de estas especies a las condiciones del medio en que se desarrollan sus poblaciones.

Por otra parte, las tres especies presentes en todas las localidades visitadas, y que muestran, asimismo, niveles de abundancia elevados ( $I$. graellsii, C. erythraea y S. fonscombii) son también las más abundantes desde hace años en las zonas dedicadas al cultivo del arroz en las marismas del Guadalquivir (Ferreras-Romero \& Soler Andrés, 1979). Cabe deducir una influencia muy grande de esta actividad agrícola en la constitución de las comunidades de odonatos presentes en los tramos inferior y medio de la cuenca del Guadiamar.

Una primera diferencia interesante entre el tramo de río aguas arriba del vertido (Gerena) y el tramo afectado más rico en especies (Sanlúcar la Mayor) aparece al considerar la presencia en el primero de las dos especies del género Platycnemis, y con valores de abundancia entre 2 y 4 , frente a la ausencia de una de ellas (P. latipes) y la bajísima densidad poblacional de $P$. acutipennis (un único individuo observado) en el caso del tramo afectado. La presencia de estas especies en la parte del río afectada por el vertido, con efectivos poblacionales similares a los encontrados en Gerena, sería indicativo de que la cuenca se está recuperando.
Información en este mismo sentido (evolución positiva) también podría ser suministrada por el género Orthetrum. De las dos especies alóctonas con origen africano, $O$. chrysostigma puede interpretarse como algo menos tolerante que $O$. trinacria, ya que algún tipo de actividad reproductora o presencia de individuos adultos recién emergidos, de la primera de estas especies, fue registrada en todas las localidades no afectadas por el vertido, en Sanlúcar la Mayor y en Aznalcázar (en esta localidad sólo un individuo); la segunda fue observada exclusivamente en tramos afectados. Asimismo, de las dos especies holomediterráneas de este género, la menos tolerante fue $O$. coerulescens, ausente incluso en los arroyos considerados posibles fuentes de recolonización; mientras que $O$. cancellatum fue frecuente y relativamente abundante en las zonas afectadas por el vertido. Es decir, podría ser reflejo de un proceso de recuperación un incremento en la distribución de $O$. coerulescens y $O$. chrysostigma.

\section{AGRADECIMIENTOS}

El presente trabajo ha sido realizado en el marco del Acuerdo específico entre la Consejería de Medio Ambiente de la Junta de Andalucía y diversas universidades andaluzas para la realización del proyecto de investigación "Evaluación del estado actual de las poblaciones de invertebrados en la cuenca del Guadiamar y seguimiento de su recuperación en el corredor ecológico".

\section{BIBLIOGRAFÍA}

AGÜERO-PELEGRÍN, M. \& M. FERRERASROMERO. 1992. Dynamics of a dragonfly community in a man-made lake of the Sierra Morena, Andalusia, southern Spain (Odonata). Opus. zool. flumin., 83: 1-7.

AGÜERO-PELEGRÍN, M.\& M. FERRERASROMERO. 1994. Dragonfly emergence from an artificial pond in the urban area of Cordoba, Andalusia, southern Spain: a possible case of intra- 
guild predation and competition between larvae. Notul. odonatol. 4 (4): 57-60.

AGÜERO-PELEGRÍN, M., M. FERRERAS-ROMERO \& P. S. CORBET. 1999. The Life Cycle of Lestes viridis (Odonata: Lestidae) in Two Seasonal streams of the Sierra Morena Mountains (southern Spain). Aquatic Insects, 21: 187-196.

ASKEW, R. R. 1988. The Dragonflies of Europe. Colchester: Harley Books.

BUCCIARELLI, I., P. A. GALLETTI \& M. PAVESI. 1983. Attuali conoscenze sul popolamento odonatologico della Sardegna. Lav. Soc. It. Biogeograf., N.S., 8: 465-544.

CARCHINI, G. \& E. ROTA. 1985. Chemico-physical data on the habitats of rheophile Odonata from central Italy. Odonatologica, 14: 239-245.

CASTELLA, E. 1987. Larval Odonata distribution as a describer of fluvial ecosystems: the Rhône and Ain rivers, France. Adv. Odonatology, 3: 23-40.

CORBET, P. S. 1999. Dragonflies: Behaviour and Ecology of Odonata. Colchester: Harley Books.

DUFOUR, C.1978. Odonates printaniers dans le Delta du Guadalquivir. Cahiers des Naturalistes, Bull. N. P., n. s. 32: 41-43.

FERRERAS-ROMERO, M. 1982. Odonatos de Sierra Morena Central (Córdoba): Aspectos faunísticos. Bol. Asoc. esp. Entom., 5: 13-23.

FERRERAS-ROMERO, M. 1988. New data on the ecological tolerance of some rheophilous odonata in Mediterranean Europe (Sierra Morena, Southern Spain). Odonatologica, 17: 121-126.

FERRERAS-ROMERO, M. 1989. Los odonatos de Andalucía (España). Análisis zoogeográfico. Misc. Zool., 13: 63-71. 1989.

FERRERAS-ROMERO, M. 1991. Preliminary data on the life history of Cercion lindeni (Sélys) in South Spain (Zygoptera: Coenagrionidae). Odonatologica, 20: 53-63.

FERRERAS-ROMERO, M. 1999. Biodiversity of rheophilous odonata in southern Spain. Odonatologica, 28: 417-420.

FERRERAS-ROMERO, M. \& A. GALLARDOMAYENCO. 1985. Los odonatos de la cuenca del río Guadiamar (Sevilla). Mediterrranea Ser. Biol., 8: 17-28.

FERRERAS-ROMERO, M. \& A. M. GARCÍA ROJAS. 1995. Life-history patterns and spatial separation exhibited by the odonates from a mediterranean inland catchment in southern Spain. Vie et Milieu, 45: 157-166.

FERRERAS-ROMERO, M. \& A. G. SOLER ANDRÉS. 1979. Odonatos de las Marismas del Bajo Guadalquivir. Aspectos faunísticos. Bol. Asoc. esp. Entom., 3: 213-218.

GALletTI, P. A. \& M. PAVESI. 1987. Considerazioni su odonati ellenici (Odonata). Atti Convegno di Zoologia Ellenica, Roma 1986: 35-37.

GRAND, D. 1994. Sur Trithemis annulata (Palisot de Beauvois, 1805) en France continentale et en Espagne du nord-est (Odonata, Anisoptera, Libellulidae). Martinia, 10: 65-71.

HAGEN, H. A. 1866. Die Neuropteren Spaniens nach Ed. Pictec's Synopsis des Neuroptères d'Espagne. Stettin Ent. Zeit., 27: 281-302.

JURZITZA, G. 1993. Libellules d'Europe. Europe centrale et méridionale. Lausanne: Delachaux et Niestlé.

OCHARAN-LARRONDO, F. J. 1987. Los Odonatos de Asturias y de España. Tesis Doctoral, Universidad de Oviedo. 983 pp.

OCHARAN-LARRONDO, F. J. 1988. Composición de la odonatofauna ibérica. Rev. Biol. Univ. Oviedo, 6: 83-93.

PRAT, N., J. TOJA, C. SOLÀ, M. D. BURGOS, M. PLANS \& M. RIERADEVALL. 1999. Effect of dumping and cleaning activities on the aquatic ecosystems of the Guadiamar River following a toxic flood. The Science of the Total Environment, 242: 231-248

PRAT, N., C. SOLÀ, M. PLANS, J. TOJA \& M. D. BURGOS. 2001. La restauración del estado ecológico del río Guadiamar. Medio Ambiente, 36: 50-55.

ROBACK, S. S. 1974. Insects (Arthropoda: Insecta). In: Pollution ecology of freshwater invertebrates. C. W. Hart \& S.L.H. Fuller (eds.): 313-376. Academic Press, London-New York.

SPONSELLER, R. A., E. F. BENFIELD \& H. M. VALET. 2001. Relationships between land use, spatial scale and stream macroinvertebrate communities. Freshw. Biol., 46: 1409-1424.

ST. QUENTIN, D. 1960. Die Odonatenfauna Europas, ihre Zusammensetzung und Herkunft. Zool. Jahrb. (Syst.), 87: 301-316. 